Revista Eletrônica de Farmácia

Eletronic Journal of Pharmacy

ISSN 1808-0804

doi $10.5216 /$ ref.v15ie.47974

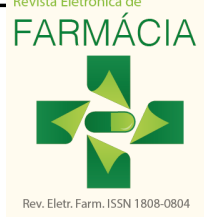

Artigo Original

\title{
Pacientes em uso crônico de prednisona: perfil Clínico e laborato- rial
}

\section{Patients in prednisone chronic use: clinical and Laboratory profile}

\section{Pacientes en uso crónico de prednisona: perfil Clínico y laboratorial}

\author{
COSTA, Ana Carolina Assis ${ }^{1}$, BOLINA, Virgínia Maria Gonçalves ${ }^{1}$, RODRIGUES, João Paulo Vilela ${ }^{2}$, REIS, Tiago \\ Marques $^{3}$, OLIVEIRA, Cláudia Di Lorenzo ${ }^{1}$, and BALDONI, André Oliveira ${ }^{1, *}$ \\ ${ }^{1}$ Universidade Federal de São João Del Rei (UFSJ), ${ }^{2}$ Faculdade de Ciências Farmacêuticas de Ribeirão Preto - Universidade de São \\ Paulo (FCFRP-USP), ${ }^{3}$ Universidade Federal de Alfenas (UNIFAL-MG) \\ *andrebaldoni@ufsj.edu.br
}

Resumo. Objetivo: Identificar a frequência e o perfil de pacientes em uso crônico de prednisona que não fazem tratamento preventivo da osteoporose induzido por corticoide e descrever as alterações laboratoriais potencialmente associadas ao uso desse medicamento. Metodologia: Realizou-se um estudo descritivo, no qual foi utilizado um roteiro de entrevista para coleta de dados, além da análise de exames bioquímicos. A coleta foi realizada de agosto de 2015 a julho de 2016. Resultados: Foram incluídos na pesquisa 34 pacientes em uso crônico de prednisona. A dose de $5 \mathrm{mg} / \mathrm{dia}$ de prednisona foi a mais prevalente

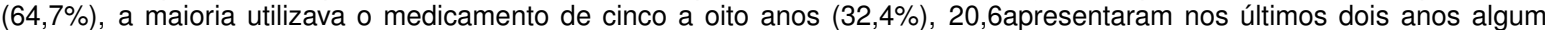
tipo de fratura óssea e $88,2 \%$ não utilizavam o medicamento para prevenção ou controle da osteoporose. Dos entrevistados, 21 realizaram os exames bioquímicos, sendo 57,1\% com níveis de lipoproteína de muita baixa densidade (LDL) acima do preconizado, 33,3\% com glicemia de jejum superior a 99mg/ dL e 28,6\% com níveis de lipoproteína de muita baixa densidade (VLDL) e triglicerídeos alterados. Discussão: $\mathrm{O}$ uso crônico de corticoide pode ser um dos fatores associados às alterações metabólicas dos pacientes. Conclusão: A frequência de pacientes que utilizam prednisona e não fazem tratamento preventivo da osteoporose é elevada, bem como a alteração dos exames laboratoriais.

Palavras-chave: Osteoporose. Corticoides. Eventos adversos.

\begin{abstract}
Objective: To identify patients frequency and profile in prednisone chronic use without preventive treatment for osteoporosis corticoid- induced and to describe the laboratory abnormalities potentially associated with prednisone use. Methods: A descriptive study was carried out with a structured questionnaire, as well as the analysis of metabolic changes. Data collection was carried out from August 2015 to July 2016. Results: The dose of 5mg/day of prednisone was the most prevalent $(64.7 \%)$, the majority used the drug for five to eight years $(32.4 \%)$, and $88.2 \%$ did not use preventive treatment for osteoporosis. Among the interviewees, 21 performed laboratory tests, and $57.1 \%$ presented low density lipoprotein (LDL) levels above the recommended, $33.3 \%$ presented fasting blood glycemia level higher than $99 \mathrm{mg} / \mathrm{dL}, 28.6 \%$ presented levels of very low density lipoprotein (VLDL) and triglycerides above of reference values. Discussion: Chronic use of corticosteroids may be one of the factors associated with patients metabolic changes. Conclusion: The frequency of patients who use prednisone and do not undergo preventive treatment for osteoporosis is high (88.2\%), as well as changing in laboratory tests.
\end{abstract}

Key-words: Osteoporosis. Corticoids. Adverse events.

Resumen. Objetivo: Identificar la frecuencia y el perfil de pacientes en uso crónico de prednisona que no hacen tratamiento preventivo para osteoporosis inducida por corticoides y describir las alteraciones en examenes laboratorialespotencialmente asociadas al uso de este medicamento. Metodología: Se realizó un estudio descriptivo utilizando un cuestionario estructurado para la recolección de datos, además del análisis de exámenes bioquímicos. La recolección fue realizada de agosto de 2015 a julio de 2016. Resultados: Fueron incluidos en el estudio 34 pacientes con uso crónico de prednisona. La dosis de $5 \mathrm{mg} / \mathrm{día}$ de prednisona fue la más prevalente $(64,7 \%)$, la mayoría utilizaba el medicamento de cinco a ocho años $(32,4 \%), 20,6 \%$ presentaron en los dos últimos años algún tipo de fractura ósea y el $88,2 \%$ no utilizaba el medicamento para prevención o control de la osteoporosis. De los entrevistados, 21 realizaron los exámenes bioquímicos, siendo $57,1 \%$ con niveles de lipoproteína de baja densidad (LDL) por encima de lo recomendado, 33,3\% con glucemia en ayunas superior a 99mg/dL y $28,6 \%$ con niveles de lipoproteína de muy baja densidad (VLDL) y triglicéridos alterados. Discusión: El uso crónico de corticoides puede ser uno de los factores asociados a las alteraciones metabólicas de los pacientes. Conclusión: La frecuencia de pacientes que utilizan prednisona y no hacen tratamiento preventivo para la osteoporosis es elevada $(88,2 \%)$, así como la alteración de los exámenes bioquímicos.

Palabras-clave: Osteoporosis. Corticoides. Efectos adversos. 


\section{Introdução}

Os corticoides são agentes esteróides que mimetizam a ação do cortisol, hormônio secretado pelas glândulas adrenais ao longo do dia com o objetivo de manter o corpo humano em homeostase, e que apresentam ação antiinflamatória e imunossupressora. Uma vez ligados ao seu receptor, os corticoides interagem com o domínio de ligação ao Ácido Desoxirribonucleico (DNA), no qual os genes são sensíveis aos seus efeitos, incluindo os genes de resposta imune e inflamatóri(1).

Amplamente utilizados na prática clínica, os corticoides são comumente prescritos para doenças de origem alérgica, inflamatória ou auto-imune, pós-transplante de órgãos e insuficiência adrenal. Recomenda-se que os pacientes em tratamento crônico (três meses ou mais) com algum fármaco desta classe sejam avaliados quanto à necessidade de indicação de tratamento preventivo da osteoporose induzida por corticoides (OPIC)(2).

Apesar da conhecida e comprovada utilidade clínica desses medicamentos, seu uso crônico está associado a importantes eventos adversos, que são manifestados em curto e em longo prazo(3). Dentre esses eventos adversos, destacam-se: hiperglicemia; diabetes mellitus; redução da massa muscular; aumento do colesterol total; diminuição da absorção intestinal e aumento da excreção urinária de Ca2+ (cálcio); aumento da reabsorção de $\mathrm{Na} 2+$ (sódio) e excreção de $\mathrm{K}+$ (potássio) pelo rim; hipertensão arterial; aumento de peso; redução da densidade mineral óssea e osteoporose(4).

A osteoporose é um distúrbio osteometabólico caracterizada pela diminuição da densidade mineral óssea, levando ao aumento da fragilidade esquelética e risco de fraturas(5). Neste contexto, é importante ressaltar que os corticoides inibem a absorção intestinal de Ca2+ (cálcio), aumentam a sua eliminação urinária e diminuem a formação osteoblástica(6).

Sendo assim, recomenda-se que pacientes em tratamento crônico com algum fármaco desta classe sejam avaliados quanto à necessidade de indicação de tratamento preventivo para osteoporose induzida por corticóides (OPIC)(2) visto que, a osteoporose aumenta em duas vezes o risco de fraturas, podendo afetar homens e mulheres que utilizam corticoides $(3,7)$.

As doses prescritas e a duração do tratamento são fatores que interferem no desenvolvimento e no grau de perda de densidade mineral óssea(8). Nesse contexto, vale destacar que o tratamento com prednisona, corticoide mais utilizado por adultos na prática clínica, por um período superior a três meses consecutivos pode favorecer o aparecimento da osteoporose, mesmo em doses baixas(9). Por isso, sugere-se que os usuários crônicos de corticoides realizem tratamento profilático para prevenção da osteoporose com vitamina $\mathrm{D}$ associada ao cálcio e/ou bisfosfonatos, bem como tratamento não farmacológico como atividade física, dieta e exposição ao sol(10,11). Além disso, é imprescindível que a monitorização laboratorial para identificação destas alterações metabólicas que podem ocorre com o uso dos corticoides $(12,13)$.

Frente ao exposto e com a escassez de estudos realizados no mundo real sobre esta temática, os objetivos do presente estudo foram identificar a prevalência e o perfil de pacientes em uso crônico de prednisona que não fazem tratamento preventivo para OPIC e descrever resultados de exames laboratoriais potencialmente associados ao uso deste medicamento.

\section{Metodologia}

Este estudo descritivo foi realizado com pacientes atendidos em farmácias que dispensam medicamentos do componente básico da Assistência Farmacêutica no Sistema Único de Saúde (SUS) do município de Divinópolis-MG, cuja população consiste em 228.643 habitantes(14). Os participantes da pesquisa foram identificados por meio do registro eletrônico das dispensações realizadas no município (Sistema Integrado de Saúde - SIS).

Para seleção dos participantes, foram utilizados os seguintes critérios de inclusão: Idade igual ou superior a 18 anos, de ambos os sexos e em uso de prednisona em qualquer dose por no mínimo 90 dias. E os critérios de exclusão foram: pacientes não aptos a responderem ao roteiro de entrevista devido à situação cognitiva (através de auto relato das unidades de saúde); e moradores de área rural ou área com elevada taxa de violência urbana.

A coleta de dados incluiu a aplicação de um roteiro de entrevista com variáveis socioeconômicas e demográficas; estilo de vida e hábitos alimentares; atividades físicas; uso de prednisona e de outros medicamentos; dados relacionados ao sexo feminino e estado geral de saúde. O roteiro de entrevista foi padronizado em um processo dividido em duas etapas: avaliação do instrumento por cinco juízes, pesquisadores da área de epidemiologia clínica e estudo piloto com dez pacientes em uso crônico de prednisona. Em ambas as etapas, alterações foram realizadas no instrumento a partir das informações obtidas no sentido de melhorar a compreensão das perguntas.

Após a padronização do instrumento de coleta de dados, iniciou-se o recrutamento de participantes. Pesquisadores (estudantes em graduação do curso de farmácia) devidamente treinados foram às residências dos usuários de corticoides e realizaram o convite para participação na pesquisa, com posterior apresentação do Termo de Consentimento Livre e Esclarecido (TCLE). As entrevistas duraram aproximadamente 30 minutos. Na sequência, foi entregue ao participante um pedido de exame, que deveria ser realizado em um laboratório terceirizado. A coleta de dados ocorreu de julho de 2015 a agosto de 2016.

Para análise de possíveis alterações laboratoriais potencialmente associadas ao uso de prednisona foram realizados 
os seguintes exames: cálcio sérico; cálcio urinário urina 24h; fosfato sanguíneo; fosfatase alcalina; glicose de jejum; sódio $(\mathrm{Na}+)$; potássio $(\mathrm{K}+)$; ácido úrico; lipidograma (triglicérides, LDL, HDL e colesterol total) e dosagem de estrógenos em mulheres. As medidas de hipertensão arterial foram de acordo com a Diretriz Brasileira de Hipertensão, 2016(15).

Os dados obtidos foram digitados no software Epiinfo versão 7, e a análise foi realizada no SPSS verão 21. Para as variáveis categóricas foram realizadas tabelas de distribuição de frequências e para as variáveis contínuas foram utilizadas medidas de tendência central.

O trabalho foi aprovado pelo Comitê de Ética em Pesquisa em Seres Humanos (CEPES) da Universidade Federal de São João Del-Rei (UFSJ), Campus Centro-Oeste Dona Lindu (CCO), número CAAE 45858315.0.0000.5545.

\section{Resultados e discussão}

Foram identificados 164 pacientes, adultos e idosos, em uso crônico de prednisona por meio do registro eletrônico das dispensações realizadas no município (Sistema Integrado de Saúde - SIS). Destes, 27 foram excluídos por residirem em áreas rurais e 22 por residirem em bairros, no município de Divinópolis - MG, com elevada taxa de violência urbana. Dos 115 potenciais participantes, 42 não foram encontrados em sua residência após duas visitas domiciliares em horários distintos, 12 não residiam no endereço disponibilizado pela Secretaria Municipal de Saúde (SEMUSA), sete não utilizavam mais o medicamento prednisona no momento da entrevista, seis foram a óbito, três não estavam aptos a responderem ao questionário e 11 pacientes recusaram a participar. Destes potenciais participantes, 34 aceitaram participar da pesquisa, sendo que apenas 21 pacientes realizaram os exames laboratoriais (Figura 1).

Com relação ao sexo dos 34 indivíduos entrevistados, prevaleceu o sexo feminino. A maioria dos participantes possuía o primeiro grau incompleto, eram aposentados ou pensionistas, possuíam casa própria, eram casados, possuíam plano privado de saúde (Tabela 1) e eram considerados idosos com a média de idade de 60 anos.

A maioria dos entrevistados relatou utilizar a prednisona na dose de $5 \mathrm{mg} /$ dia. Observou-se ainda que um terço dos participantes utilizava o medicamento por um período de cinco a oito anos (Tabela 2).

A maioria dos pacientes utilizava o medicamento prednisona para tratar artrite reumatóide $(55,9 \%)$ e transplante de órgãos $(8,8 \%)$. Cerca de $60 \%$ dos entrevistados relataram exposição diária ao sol por, pelo menos, 30 minutos e pouco mais da metade relataram realizar atividade física regularmente (Figura 2). Um em cada cinco participantes $(20,6 \%, n=7)$ havia realizado, até a data da entrevista, exame para diagnóstico ou controle da osteoporose, 88,2\% $(\mathrm{n}=30)$ não utilizavam medicamento para prevenção e dos

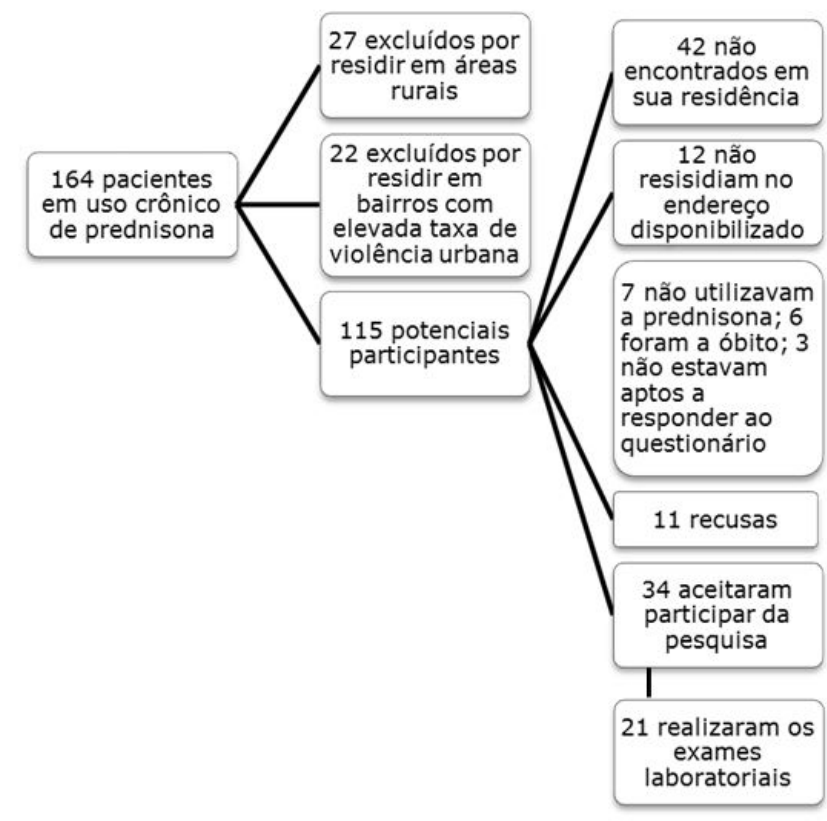

Figura 1. Fluxo de recrutamento e inclusão dos participantes do estudo, que fazem uso crônico de prednisona, 2015-2016.

\begin{tabular}{|c|c|c|}
\hline Variável & $n=34(\%)$ & $\mathbf{N}=\mathbf{2 1}(\%)$ \\
\hline \multicolumn{3}{|l|}{ Sexo } \\
\hline Feminino & $23(67,6)$ & $15(71,4)$ \\
\hline Masculino & $11(32,4)$ & $6(28,6)$ \\
\hline \multicolumn{3}{|l|}{ Idade } \\
\hline Menor ou igual a 60 anos & $15(44,1)$ & $11(52,4)$ \\
\hline Maior que 60 anos & $19(55,9)$ & $10(47,6)$ \\
\hline \multicolumn{3}{|l|}{ Escolaridade } \\
\hline Nunca frequentou a escola & $1(2,9)$ & $1(4,7)$ \\
\hline Ensino fundamental incompleto & $18(53,0)$ & $14(66,7)$ \\
\hline Ensino fundamental completo & $6(17,6)$ & $2(9,5)$ \\
\hline Ensino médio incompleto & $2(5,9)$ & $1(4,7)$ \\
\hline Ensino médio completo & $3(8,8)$ & $3(14,4)$ \\
\hline Superior incompleto & 0 & 0 \\
\hline Superior completo & $4(11,8)$ & 0 \\
\hline \multicolumn{3}{|l|}{ Situação trabalhista } \\
\hline Empregado ou funcionário público & $3(8,8)$ & $2(9,5)$ \\
\hline Doméstico & $4(11,8)$ & $2(9,5)$ \\
\hline Conta própria e outros & $6(17,6)$ & $3(14,4)$ \\
\hline Trabalhador não remunerado & $2(5,9)$ & $1(4,7)$ \\
\hline Aposentado ou pensionista & $19(55,9)$ & $13(61,9)$ \\
\hline \multicolumn{3}{|l|}{ Estado civil } \\
\hline Solteiro & $6(17,6)$ & $6(28,6)$ \\
\hline Casado & $20(58,9)$ & $14(66,7)$ \\
\hline Viúvo & $3(8,8)$ & $1(4,7)$ \\
\hline Separado & $4(11,8)$ & 0 \\
\hline União estável & $1(2,9)$ & 0 \\
\hline \multicolumn{3}{|l|}{ Casa } \\
\hline Própria & $28(82,3)$ & $17(80,9)$ \\
\hline Alugada & $2(5,9)$ & $1(4,7)$ \\
\hline Casa de familiares & $4(11,8)$ & $3(14,4)$ \\
\hline \multicolumn{3}{|l|}{ Plano privado de saúde } \\
\hline Sim & $18(52,9)$ & $14(66,7)$ \\
\hline
\end{tabular}

Tabela 1. Características socioeconômicas demográficas e econômicas da população total estudada $(n=34)$ e dos participantes que realizaram os exames laboratoriais $(n=21), 2015-2016(n=34)$. 


\begin{tabular}{lll}
\hline Variável & $\mathbf{n = 3 4}(\mathbf{\%})$ & $\mathbf{n = 2 1}(\mathbf{\%})$ \\
\hline Dose de prednisona/ dia & $22(64,7)$ & $13(61,9)$ \\
$5 \mathrm{mg}$ & $4(11,8)$ & $3(14,4)$ \\
$10 \mathrm{mg}$ & $7(20,6)$ & $4(19,0)$ \\
$20 \mathrm{mg}$ & $1(2,9)$ & $1(4,7)$ \\
Não soube informar & & \\
Período de tempo de uso & $9(26,4)$ & $3(14,4)$ \\
De 1 a 4 anos & $11(32,4)$ & $7(33,3)$ \\
De 5 a 8 anos & $4(11,8)$ & $2(9,5)$ \\
De 9 a 12 anos & $7(20,6)$ & $7(33,3)$ \\
De 13 anos ou mais & $3(8,8)$ & $2(9,5)$ \\
Não sabe quanto tempo &
\end{tabular}

Tabela 2. Dose e duração do tratamento entre os usuários crônicos de prednisona, população total estudada $(n=34)$ e participantes que realizaram os exames laboratoriais $(n=21) 2015-2016(n=34)$.

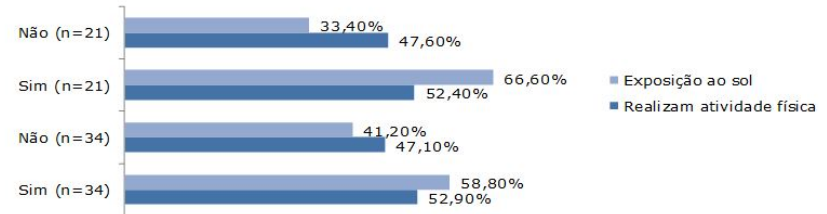

Figura 2. Medidas não farmacológicas de controle e prevenção da osteoporose realizada pelos pacientes em uso crônico de prednisona, pacientes totais $(\mathrm{n}=34)$ e os que realizaram os exames laboratoriais do presente estudo $(n=21)$ e 2015-2016 $(n=34)$.

pacientes com a faixa etária de 60 anos ou mais, 20,6\% $(\mathrm{n}=7)$ tiveram algum tipo de fratura nos últimos dois anos, visto que a maioria utilizava o corticoide para o tratamento de artrite reumatoide e destes apenas dois realizavam profilaxia para a osteoporose.

É importante destacar que, entre os 21 pacientes que realizaram os exames laboratoriais solicitados, todos apresentaram, pelo menos, um resultado alterado. Utilizando-se como parâmetro os dez exames investigados, nove apresentaram alterações em relação aos valores de referência. $\mathrm{O}$ único exame em que não foram encontradas alterações foi a dosagem de potássio (Tabela 3 ). Em relação às medidas de pressão arterial, os participantes não apresentaram valores acima do preconizado pela Diretriz Brasileira de Hipertensão, sendo que destes, 47,0\% $(n=16)$ estavam em tratamento com medicamentos anti-hipertensivos(15).

A maior parte dos usuários de prednisona eram mulheres, resultado semelhante ao encontrado por Cardoso e Rosendo (2012)(16). Este resultado pode ser justificado pela doença mais comumente observada no estudo, à artrite reumatoide $(55,9 \%)$, que ocorre predominantemente em mulheres. Em relação a esta morbidade e aos resultados sobre o tratamento preventivo da osteoporose encontrados neste estudo, é válido destacar que o "Protocolo Clínico e Diretrizes Terapêuticas da Artrite Reumatoide (2013)" preconiza que a suplementação com cálcio e vitamina D deve ser considerada no caso de uso de corticoide por mais de três meses(17). E os achados sugerem que esse preceito clínico não tem sido

\begin{tabular}{|c|c|c|c|c|c|}
\hline \multicolumn{3}{|l|}{ Exame } & $\begin{array}{c}\text { Abaixo do } \\
\text { valor de referência } \\
\text { n (\%) }\end{array}$ & $\begin{array}{l}\text { Norm } \\
\text { al } \\
\mathrm{n}(\%)\end{array}$ & $\begin{array}{c}\text { Acima do } \\
\text { valor de } \\
\text { referência } \\
n(\%)\end{array}$ \\
\hline \multicolumn{3}{|l|}{ Glicemia } & 0 & $\begin{array}{c}14 \\
(66,7)\end{array}$ & $7(33,3)$ \\
\hline \multicolumn{3}{|l|}{ Potássio } & 0 & $(100)^{21}$ & 0 \\
\hline \multicolumn{3}{|l|}{ Sódio } & $1(4,8)$ & $\begin{array}{c}20 \\
(95,2)\end{array}$ & 0 \\
\hline \multicolumn{3}{|c|}{ Ácido úrico (06 homens) } & 0 & $\begin{array}{r}5 \\
(83,33)\end{array}$ & $1(16,67)$ \\
\hline \multicolumn{3}{|c|}{ Ácido úrico (15 mulheres) } & $1(6,66)$ & $9(60)$ & $5(33,34)$ \\
\hline \multicolumn{3}{|l|}{ HDL } & $1(4,8)$ & ${ }_{(85,7)}^{18}$ & $2(9,5)$ \\
\hline \multicolumn{3}{|l|}{ LDL } & 0 & $(42,9)^{9}$ & $12(57,1)$ \\
\hline \multicolumn{3}{|l|}{ VLDL } & 0 & $\begin{array}{c}15 \\
(71,4)\end{array}$ & $6(28,6)$ \\
\hline \multicolumn{3}{|c|}{ Colesterol total } & 0 & $\begin{array}{c}13 \\
(61,9)\end{array}$ & $8(38,1)$ \\
\hline \multicolumn{3}{|c|}{ Triglicérides } & $13(61,9)$ & $(9,5)^{2}$ & $6(28,6)$ \\
\hline \multicolumn{3}{|c|}{ Cálcio urinário } & $7(33,3)$ & $\begin{array}{c}12 \\
(57,1)\end{array}$ & $2(9,6)$ \\
\hline \multicolumn{3}{|c|}{ Cálcio sérico } & $2(9,5)$ & $\begin{array}{c}19 \\
(90,5)\end{array}$ & 0 \\
\hline \multicolumn{3}{|c|}{ Fosfato sanguíneo } & 0 & $\begin{array}{r}20 \\
(95,2)\end{array}$ & $1(4,8)$ \\
\hline $\begin{array}{l}\text { Fosfatase } \\
\text { homens) }\end{array}$ & & & 0 & & 0 \\
\hline $\begin{array}{l}\text { Fosfatase } \\
\text { mulheres) }\end{array}$ & alcalina & (14 & 0 & $\begin{array}{l}(100) \\
12 \\
(85,7)\end{array}$ & $2(14,3)$ \\
\hline
\end{tabular}
Lipoproteína de densidade muito baixa.

Tabela 3. Exames laboratoriais dos pacientes em uso crônico de prednisona, 2015-2016 $(\mathrm{n}=21)$.

colocado em prática.

Os corticoides orais, mesmo em doses baixas, podem aumentar a incidência de fraturas em pouco tempo de uso do medicamento. (18) E neste estudo a prevalência de pacientes em uso de prednisona na dose de $5 \mathrm{mg}$ foi de $64,7 \%$ e 32,4\% utilizavam o fármaco por um período de 5 à 8 anos.

Apenas 20,6\% dos entrevistados haviam realizado, até a data da entrevista, exame para diagnóstico ou controle da osteoporose e $88,2 \%$ não utilizavam nenhum medicamento para prevenção da osteoporose. Esta situação sugere que os prescritores não estão seguindo protocolo de prevenção de osteoporose, uma vez que é recomendada a prevenção da osteoporose nos pacientes em uso crônico de corticoides(19). Além disso, é fundamental a adoção de medidas não farmacológicas como dieta rica em cálcio, exposição ao sol e prática de atividades físicas. No entanto, apenas $53 \%$ dos entrevistados relataram praticar exercício físico. A literatura evidencia que esta medida não farmacológica contribui para aumento da densidade mineral óssea, para redução do risco de queda e fratura óssea(13). Além disso, mais de $40 \%$ dos entrevistados referiram que não se expõem regularmente ao sol ou realizam alguma atividade sob a luz natural, que é uma medida essencial para ativação de vitamina $\mathrm{D}(11)$. 
Em relação aos exames, $57,1 \%$ dos pacientes apresentaram níveis de LDL acima do preconizado (>100mg/dL), $33,3 \%$ dos pacientes apresentaram glicemia de jejum superior a $99 \mathrm{mg} / \mathrm{dL}$. E ainda, $28,6 \%$ dos pacientes em uso crônico de corticoides apresentaram níveis de VLDL (lipoproteína de muito baixa densidade) e triglicerídeos acima dos valores referência. Das 14 mulheres que realizaram o exame de fosfatasse alcalina duas $(9,5 \%)$ apresentaram valores alterados. Na presença de osteoporose esta enzima apresenta valores elevados, visto que ela é produzida pelos osteoblastos durante a formação óssea osteoblástica(20,21). Estas alterações reforçam a necessidade de rigorosa avaliação clinica e laboratorial exigida no acompanhamento destes pacientes, no sentido de identificar, evitar e tratar eventuais reações adversas desta terapia.

Em relação aos sete $(33,3 \%)$ pacientes apresentaram valores de glicemia de jejum acima do preconizado. É valido destacar que quatro deles relataram possuir Diabetes Mellitus. Desta forma, é importante enfatizar que os níveis glicêmicos podem estar alterados devido a causas multifatoriais, como presença de comorbidades. No entanto, no contexto do uso dos corticoides, é importante considerar que estes medicamentos reduzem a captação e utilização da glicose e aumenta a gliconeogênese, além de aumentar o catabolismo e reduzir o anabolismo protéico, podendo, à vista disso, ocasionar hiperglicemia e Diabetes Mellitus(22).

Em relação ao perfil lipídico, $57,1 \%$ dos participantes apresentaram níveis de LDL (lipoproteínas de baixa densidade) acima dos valores preconizados (Menor que 100 $\mathrm{mg} / \mathrm{dL}$ ). Um estudo realizado em 2013, por Oliveira e colaboradores(23), encontrou valores de LDL significativamente maiores em pacientes em tratamento de hiperplasia adrenal congênita (HAC) com glicocorticóides quando comparados à pacientes sem tratamento ou com baixa adesão a ele. E ainda, de acordo com Kuronie e colaboradores (2010)(24), diferentes doses de glicocorticóides podem interferir sobre o perfil lipídico dos usuários, ocasionando aumento significativo nos valores de colesterol total, LDL e HDL. Ainda em relação ao perfil lipídico, os níveis de VLDL (lipoproteína de muito baixa densidade) e triglicerídeos apresentaram-se acima dos valores de referência em $28,6 \%$ dos pacientes em uso crônico de corticoides. Esses achados corroboram as evidências de que o uso crônico de corticoides está associado à elevação de lipoproteínas (VLDL e LDL), consequientemente um aumento dos níveis séricos de colesterol total e triglicérides, produção de ácidos graxos e atividade da lípase endotelial hepática(25).

Por fim, diante da dificuldade de encontrar pessoas que se comprometessem a participar do estudo e a realizar todos os exames, este estudo limitou-se a efetivamente 34 entrevistados e 21 deles realizaram exames. Muito embora os resultados tenham sido clinicamente relevantes, o tamanho amostral reduzido permitiu apenas uma análise descritiva dos resultados, não sendo possível identificar variáveis explicativas para a variável resposta do estudo.

Frente aos resultados encontrados e os dados disponíveis na literatura sugere-se que os exames apresentados a seguir sejam padronizados e inseridos nos protocolos clínicos da

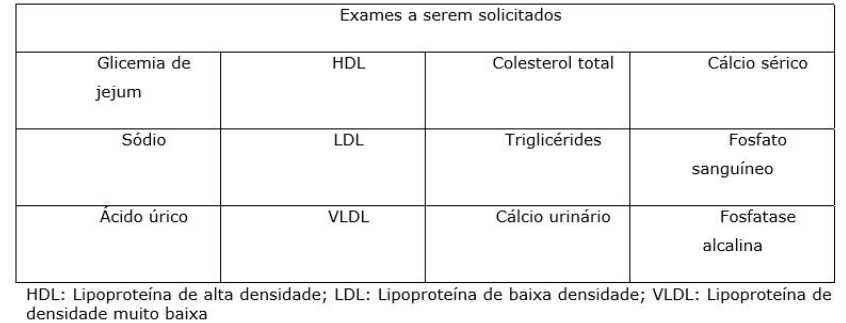

Quadro 1: Exames laboratoriais que devem ser, regularmente, solicitados a pacientes em uso crônico de corticoides.

atenção primária para que, regularmente, sejam solicitados a pacientes em uso crônico de corticoides (quadro 1).

\section{Conclusão}

Os resultados evidenciaram elevada prevalência de pacientes em uso crônico de prednisona que não utilizam medicamento para prevenção da osteoporose $(88,2 \%)$. Em relação às medidas para prevenção da osteoporose, 58,8\% dos pacientes se expõem ao sol ou realizam alguma atividade sobre a luz natural e 52,9\% realizam atividade física. Dentre os participantes que realizaram os exames bioquímicos, todos apresentaram pelo menos, um resultado alterado. Esses resultados evidenciam a necessidade de ações educativas aos profissionais de saúde, visando orientá-los em relação ao acompanhamento e o monitoramento destes pacientes, uma vez que é elevada a prevalência de valores de exames laboratoriais fora do preconizado.

\section{Agradecimentos}

À Secretaria Municipal de Saúde do Município de Divinópolis - MG (SEMUSA) pelo apoio institucional e à Fundação de Amparo à Pesquisa do Estado de Minas Gerais (FAPEMIG) pelo apoio financeiro.

\section{Referências}

1. Straub R, Cutolo M. Glucocorticoids and chronic inflammation.Rheumatology 2016; (55).

2. Grossman JM, Gordon R, Ranganath VK, Deal C, Caplan L, Chem W. et al. American College of Rheumatology 2010 recommendations for the prevention and treatment of glucocorticoid-induced osteoporosis. ArthritisCare Res (Hoboken) 2010; (62):1515-1526.

3. Album JM, Lévesque LE, Gershon AS, Liu G, Cadarette SM.Glucocorticoid-induced osteoporosis management among seniors, by year, sex, and indication, osteoporosis, July 2015; 1996-2012.

4. MICROMEDEX® 2.0. 2017. Disponível em: http://www-micromedexsolutionscom.ez67.periodicos. capes.gov.br/micromedex2/librarian/. [acesso em janeiro de 2017]. 
5. Ukert BP. Glucocorticoid and drugs-induced osteoporosis. In: Favus MJ, editor. Primer on the metabolic bone diaseases and disorders of mineral metabolism.LippincottRavenPublishers, Philadelphia, 1996; 278-282.

6. Borba V.; Castro M. Osteoporose induzida por glicocorticóide. Arq Bras Endocrinol Metab, 1999; 43(6). 7. National Osteoporosis Foundation.Clinician's guide to prevention and treatment of osteoporosis. Washington, DC: NationalOsteoporosis Foundation; 2010.

8. Emkey GR, Epstein S. Best practice \& Research clinical endocrinology \& metabolism. Secondary osteoporosis: Pathophysiology \& diagnosis, New York 2014; (28):911-935.

9. Fiehn C. Glucocorticoid therapy in rheumatoid arthritis - contra.Dtsch med Wochenschr 2016; 141(22):1651.

10. Pereira RMP, Paula AP, Zerbini C, Domiciano DS, Gonçalves H, Danowski J. Osteoporose induzida por glicocorticóide. Associação médica brasileira e conselho federal de medicina, Set. 2011.

11. Cesareo R, Iozzino M, D’onofrio L, Terrinoni I, Maddaloni E, Casini A et al. Effectiveness and safety of calcium and vitamina $\mathrm{D}$ treatment for postmenopausal osteoporosis. Minerva Endocrinol 2015; 40(3):231-237.

12. Kanis JA, Mccloskey EV, Johansson H, Cooper C, Rizzoli R, Reginster JY. Europeanguidance for thediagnosisand management osteoporosis in postmenopausal women.OsteoporosInt 2013; (24):23-57.

13. BRASIL. Ministério da Saúde. Secretaria de Atenção à Saúde. Portaria 451 - Aprova o Protocolo Clínico e Diretrizes Terapêuticas da Osteoporose. Brasília (DF), 2014. Disponível em: http://portalarquivos.saude.gov.br/images/pdf /2014/junho/10/Republica-o-Portaria-n-451-de-09-dejunho-de-2014-atual.pdf. [Acesso em 17 de dezembro de 2016].

14. IBGE - Instituto Brasileiro de Geografia e Estatística - 2014. Disponível em http://www.cidades.ibge.gov.br /xtras/perfil.php?lang=codmun=312230. [Acesso em $17 \mathrm{de}$ junho de 2015].

15. Malachias MVB, Souza WKSB, Plavnik FL, Rodrigues CIS, Brandão AA, Neves MFT, et al. Diretrizes Brasileiras de Hipertensão. Ver Bras Hipertens 2016; 107(3). Disponível em: http://www.scielo.br/pdf/abc/ v107n3s3/0066-782X-abc-107-03-s3-0067.pdf. [Acesso em 15 de novembro de 2016].
16. Cardoso R, Rosendo A. Adesão dos pacientes ao tratamento para osteoporose em Tubarão-SC. Arq. Catarin.Med 2012; 41(2).

17. BRASIL. Ministério da Saúde Secretaria de Atenção à Saúde PORTARIA N ${ }^{\circ} 710$, DE 27 DE junho DE 2013. Aprova o Protocolo Clínico e Diretrizes Terapêuticas da Artrite Reumatoide. Disponível em: http://bvsms.saude. gov.br/bvs/saudelegis/sas/2015/ prt0996_30_09_2015.html [Acesso em 15 de novembro de 2016].

18. Krasselt M, Baerwald C. Glukokortikoid - induzierteosteoporose: ein Update. DtschmedWochenschr 2016; 141(05):352-357.

19. Pereira RMR.; Carvalho JF.; Paula AP.; Zerbini C.; Domiciano DS.; Gonçalves H.Diretrizes para prevenção e tratamento da osteoporose induzida por glicocorticóide. Ver Bras Reumatol, São Paulo 2012; 52(4).

20. Patrício JP, Oliveira P, Faria MT, Pérez MB, Pereira J. Osteoporose Induzida por Corticoides. Arquivos de Medicina: Arqui Med, Porto 2006; 20(5/6):173-178.

21. Vieira JGH. Considerações Sobre os Marcadores Bioquímicos do Metabolismo Ósseo e sua Utilidade Prática. Endrocrinol Metab, São Paulo 1999; 43(6): 415-422.

22. Battastini AMO; Bernardi A; Bavaresco L,. Glicocorticoides: usos clássicos e emprego no tratamento do câncer. Infarma, Porto Alegre 2005; 17(7/9):58-60.

23. Oliveira LMB, Junior JADF, Silva DN, Lago R, Toralles MBP. Valores elevados de leptina e LDL-colesterol em pacientes com hiperplasia adrenal congênita bem controlados. Arq Bras Endocrinol Metab, São Paulo 2013; 57(5):354-359.

24. Kuroki Y, Kaji H, Kawano S, Kanda F, Takai Y, Kajikawa M, et al. Prospectiveshort-term effects of glucocorticoid treatment on glucoseand lipid metabolism in Japanese. Intern Med 2010; 49(10):897-902.

25. Taskinem MR, Nikkila EA, Pelkonem R, Sane T. Plasma lipoproteins, lipolytic enzymes, and very low density lipoprotein triglyceride turnover in cushing 's syndrome. J Clin. Endocrinol. Metab. Baltimore 1983; 57(3):619-626. 